\title{
Excision margins in squamous cell carcinoma of the tongue: A retrospective audit and review of the literature
}

\author{
Miriam Thake ${ }^{1}$, Athanasis Kalantzis ${ }^{2}$ \\ ${ }^{1}$ Oxford University Medical School, Oxford Radcliffe Hospitals, Oxford University, Oxford, UK \\ ${ }^{2}$ Maxillofacial Surgery, Oxford Radcliffe Hospitals, Oxford, UK \\ Email: miriam@thakes.co.uk
}

Received 21 January 2013; revised 28 February 2013; accepted 10 March 2013

\begin{abstract}
The incidence of close and involved tongue resection margins for squamous cell carcinoma (SCC) were reviewed with the aim to identify any possible need for change in the surgical approach to glossectomies. The histopathological reports of 101 partial glossectomies for SCC between 2006 and 2012 were retrospectively reviewed. Results: Overall 52 (51.5\%) patients had one or more close or involved margin and 9 (8.9\%) had both close and involved margins. 42 (41.5\%) patients had close margins and $11(10.9 \%)$ had involved margins. The inferior/lateral muscoal margin was most frequently close/involved (32\%) followed by deep margin (27\%). The anterior margin was least close/involved (5\%). The posterior and superior/medical margins were close/involved in $12 \%$ and $11 \%$ of cases respectively. Conclusions: $52.5 \%$ of patients had close or involved margins following surgery, potentially requiring further treatment to avoid an increased risk of tumour recurrence and the associated increase in morbidity and mortality. The inferior/lateral and deep margins were most frequently involved possible due to the anatomical difficulties visualising and dissecting these margins. The potential explanations for these disparities and possible solutions are discussed.
\end{abstract}

Keywords: Squamous Cell Carcinoma; Tongue Cancer; Partial Glossectomy; Excision Margins

\section{INTRODUCTION}

The involvement of tumour at surgical margins after resection of squamous cell carcinoma from the tongue suggests a poor prognosis. Tumour margin involvement has an increased probability of local recurrence post glossectomy. The reported rates are $18 \%-23.5 \%$ and the majority of recurrences occur within the first year of surgery [1-4]. Furthermore, the high rate of local tumour recur- rence as a consequence of narrow margins may not decrease with the addition of adjuvant radiotherapy [5]. It is therefore not surprising that most studies show a negative impact of close or involved margins on disease-specific survival [1-3,6-10].

The impact of positive margins on local recurrence and survival highlights the need for adequate clearance margins during partial glossectomies. The current aim of surgical tumour excision is a microscopic margin of at least $5 \mathrm{~mm}$, requiring an intraoperative margin of at least $1 \mathrm{~cm}$ [11]. However, the size of the margin taken is often limited by a number of different factors. Firstly, large margins may involve the sacrifice of a significant proportion of the tongue, floor of mouth or mandible, and this may cause a substantial impact on subsequent quality of life. The tongue is vital for both deglutition and speech, and removal of large areas of the tongue can result in the decreased ability to swallow and articulate. The size of the margin that can be taken is also influenced by the possibility of damaging surrounding anatomical structures. For example, the deep margin often lies close to the contralateral lingual artery, sacrifice of which may necessitate a nearly total glossectomy. Similarly the inferior/lateral margin may be limited by the mandibular periosteum meaning further margin clearance can only achieved by some form of mandibulectomy. Surgeons performing glossectomies are therefore faced with the delicate task of balancing the need to achieve adequate tumour margins with the desire to retain function and quality of life and limit the cosmetic defect.

It is therefore important to gather information about the success of current surgical resection techniques for squanous cell carcinoma of the tongue in order to identify any need for change in the surgical approach to glossectomies. The aim of this study was to review the tumour margins following 101 consecutive partial glossectomies to assess whether sufficient margins are consistently being taken and, if not, whether particular margins are more commonly involved than others. 


\section{METHODS}

The histopathology records of all patients with T1/T2 SCC of the tongue who underwent partial glossectomy at the John Radcliffe Hospital, Oxford between 2006 and 2012 were reviewed. One hundred and one patients were identified from the operating theatre log book and for these patients the superior/medial, inferior/lateral, anterior, posterior and deep/muscle margins were recorded. Data was retrieved from the patient's electronic case records and was taken from the reports independently of the surgical team and histopathologists. Any patients with tumour extending beyond the midline or to other anatomical structures or with dysplasia noted in the margins were excluded.

The UK guidelines, based on recommendations from the Royal College of Pathologists were used to define "clear", "close" and "involved" tumour margins. These guidelines define margins less than $1 \mathrm{~mm}(<1 \mathrm{~mm})$ as involved, margins equal to $1 \mathrm{~mm}$ and up to $4.9 \mathrm{~mm}(\geq 1$ $\mathrm{mm}$ to $<5 \mathrm{~mm}$ ) as close and margins of $5 \mathrm{~mm}$ or greater as clear $(\geq 5 \mathrm{~mm})$ [11].

\section{RESULTS}

Overall 42 patients $(41.5 \%)$ had close margins and a further $11(10.9 \%)$ had involved margins (Table 1). Some patients had more than 1 close or involved margin and 9 (8.9) patients had both close and involved margins. The inferior/lateral muscoal margin was the most frequently close or involved (32 of 101 [31.7\%]) followed by deep margin (27 of 101 [26.7\%]). The anterior margin was the least commonly close or involved (6 of 101 [5.9\%]). The posterior and superior/medical margins were close or involved in 12 of $101(11.9 \%)$ and 11 of $101(10.9 \%)$ of cases respectively (Table 2 ).

Considering only the involved margins defined as $<1$ $\mathrm{mm}$, the interior/lateral margin was still the most frequently affected with 6/101 (5.9\%) involved, followed by the posterior margin $(5 / 101$ [5.0\%]). The deep margin was involved in $(3 / 101[3.0 \%])$ and the superior/medial and anterior margins were involved in $1 / 101(1 \%)$ and $2 / 101(2 \%)$ respectively.

\section{DISCUSSION}

In surgical excision of SCC of the tongue, the surgeon is often faced with a significant discrepancy between the tumour-free margins thought to be achieved in-situ and those reported from histopathological analysis. This is primarily due to tissue shrinkage, which occurs at various stages following the excision and processing of the tumour [12,13]. Mistry et al., found that mean shrinkage of the tongue margins was $23.5 \%$, with $\mathrm{T} 1 / 2$ tumours shrinking significantly more than T3/4 tumours [13]. Johnson et al., reported average tongue mucosal margin
Table 1. Total number of patients with close, involved and close/involved margins.

\begin{tabular}{cc}
\hline Excision margin & $\begin{array}{c}\text { Number of patients with margin } \\
\text { involved }\end{array}$ \\
\hline Close & $42 / 101(41.5 \%)$ \\
Involved & $11 / 101(10.9 \%)$ \\
Close or involved & $52 / 101(51.5 \%)$ \\
Close and involved & $9 / 101(8.9 \%)$ \\
\hline
\end{tabular}

shrinkage of $30.7 \%$ while the deep/muscle margin shrank by $34.5 \%$, with the greatest proportion of shrinkage occurring immediately upon excision [12]. Therefore, most surgeons aim for an in-situ excision margin of $10 \mathrm{~mm}$ during glossectomies, in order to achieve histological clearance of $5 \mathrm{~mm}$. Yuen et al., advocate even wider excision margins, as the larger the area of macroscopically normal tissue around the tumour, the more likely it is that microscopic extensions are removed [14]. Alternatively, Kakarala et al., suggest that the use of different types of surgical techniques can have an impact on the level of tissue distortion at the surgical margin, with steel scalpel causing the least margin disruption and harmonic scalpel creating less distortion than monopolar electrosurgery [15].

There is some evidence that the involvement of the deep margin in particular is related to increased rates of local recurrence of squamous cell carcinoma of the tongue [1]. Recurrence at this site is also more difficult to identify at an early stage during post treatment surveillance and more difficult to treat with further surgery, especially if the tongue has been reconstructed with free tissue transfer. It is therefore of note that in our audit, the deep margin was the second most frequently close/involved margin. There are a number of potential explanations for this high incidence of incompletely excised deep margins including the inability to visualise this margin, fear of damaging the contralateral lingual artery and a desire to maintain as much muscle as possible in order to preserve tongue function. It is also possible that this result is an artefact of the histological process as the deep margin has been shown to shrink by the greatest proportion [12]. The use of diathermy for tumour resection may also increase the incidence of "apparent" close margins, due to the destructive nature of this technique compared to cold steel excision, but this is likely to affect all margins equally. Diathermy excision with a Colorado needle was used in the majority of our cases.

The inferior/lateral margin was also commonly close or involved in our study. This may be because the margin often requires resection into the posterior floor of mouth or lingual alveolar mucosa making it more difficult to visualise and access compared to other margins. In some of these cases however, the lateral margin may actually 
Table 2. Percentage of close, involved and close/involved cases for each margin.

\begin{tabular}{cccccc}
\hline \multirow{2}{*}{ Excision margin } & \multicolumn{3}{c}{ Margin } \\
\cline { 2 - 6 } & Inferior/lateral & Superior/medial & Posterior & Anterior & Deep \\
\hline Close & $26 / 101(25.7 \%)$ & $10 / 101(9.9 \%)$ & $7 / 101(6.9 \%)$ & $4 / 101(4.0 \%)$ & $24 / 101(24.0 \%)$ \\
Involved & $6 / 101(5.9 \%)$ & $1 / 101(1.0 \%)$ & $5 / 101(5.0 \%)$ & $2 / 101(2.0 \%)$ & $3 / 101(3.0 \%)$ \\
Close/Involved & $32 / 101(31.7 \%)$ & $11 / 101(10.9 \%)$ & $12 / 101(11.9 \%)$ & $6 / 101(5.9 \%)$ & $27 / 101(26.7 \%)$ \\
\hline
\end{tabular}

coincide with the mandibular periosteum. Such close margins may be considered "acceptable" as an uninvolved periosteum may act as a barrier, negating the need for mandibular resection in an effort to achieve at least 5 $\mathrm{mm}$ of clearance.

Many techniques have been used to try to reduce the frequency of close or involved margins. Firstly, a number of intra-operative measures have been developed in an attempt to achieve primary tumour clearance. Intraoperative frozen sections have traditionally been used for assessment of excision [16,17]. However, one margins found to be positive with frozen sections and subsequently rendered negative with further resection may still have an increased risk of local recurrence, especially if radiotherapy is not used postoperatively [18] and the use of frozen sections is therefore not popular in all units. Gauthier et al., described a Mohs-like technique for excision of tongue SCC with a $1-5 \mathrm{~mm}$ margin and described no tumour recurrences, although their follow-up was short [19]. Intra-oral ultrasound for the assessment of tumour thickness and of intra-operative tumour clearance has also been used with some success $[20,21]$. The intra-operative use of Lugol's iodine staining has also been reported to assist local clearance at the mucosal margins and reduce recurrence [22]. Kurita et al., reported that intra-operative tissue staining and examination under a microscope permits visual inspection of a central section of the surgical specimen providing an accurate assessment and therefore better control of deep margins in oral cancer surgery [23]. Furthermore, Keereweer et al., have recently used animal models to suggest that it may be possible to use intra-operative visualization techniques with near-infrared (NIR) fluorescence optical imaging to provide real-time image-guided surgery [24].

Further solutions to the problem of close margins could also include improved surgical techniques. Evidence for this comes from Lee et al., who demonstrated a positive relationship between patient survival and surgeon caseloads. They suggested that the treatment strategies adopted by high-volume surgeons should be analysed and utilised more widely although it is unclear from this study whether the more experienced surgeons achieve a higher proportion of clear surgical margins [25]. Surgical techniques providing improved access (such as mandibulotomy or pull through technique) and in continuity resection (where tongue, floor of mouth and neck specimen are removed en block) are employed by some surgeons to achieve better margin clearance, but evidence to support these is lacking. Others have noted that accurate and extensive imaging before surgery can reduce the frequency of involved margins [26,27].

In a cohort of 200 patients with oral SCC, Sutton et al., reported close or involved surgical margins in $46.5 \%$ [4]. Interestingly, they found that these close or involved margins were highly related to histological indicators of aggressive disease such as lymphovascular and perineural invasion. They concluded that close surgical margins in oral SCC could be regarded as an indicator of aggressive disease and therefore surgical technique may not be the primary determinant of margins status.

The importance of distinguishing between close and involved margins has been emphasised by some $[28,29]$. In a retrospective review of 192 patients with oral and oropharyngeal cancer Wong et al. found that only involved margins were associated with local recurrence [29]. Overall, $12 \%$ of patients had involved and a further $55.7 \%$ had close $(1-5 \mathrm{~mm})$ margins. Sixty of the 107 patients with close margins received no adjuvant therapy, and of these only 5 had local recurrence. Close margins did have an adverse association with disease-free survival, however, the cut-off point was $\leq 1.6 \mathrm{~mm}$, emphasising the arbitrary choice, by most researchers, of $5 \mathrm{~mm}$ as the cut-off point, and recommending redefining close margins as $1-2 \mathrm{~mm}$.

Ultimately, even if there are tumour free margins at pathology, it is impossible to guarantee that there will be no recurrence. The microscopic presence of tumour cells within the margin is not the only predictor of recurrence and a number of other factors have been associated with increased rates of local recurrence including the histological pattern of invasion, site and stage of the tumour, perineural invasion and depth of invasion $[1,17,30]$. Methods of molecular and immunocytochemical detection of minimal residual cancer and field cancerisation have also been developed and may help identify patients at risk of developing loco-regional or distant recurrence [31].

However, careful surgery to achieve negative resection margins remains the most important factor in the effort to 
improve local control of tongue cancer and diseasespecific survival. Our results suggest that greater care needs to be taken, particularly at the deep/muscle and inferior/lateral margins, to ensure adequate resection. Certain access procedures and adjunct techniques such as intra-operative ultrasound may be of some help, but further studies are needed to demonstrate this.

\section{REFERENCES}

[1] Kirita, T., Okabe, S., Izumo, T. and Sugimura, M. (1994) Risk factors for the postoperative local recurrence of tongue carcinoma. Journal of Oral and Maxillofacial Surgery, 52, 149-154.

[2] Woolgar, J.A., Rogers, S., West, C.R., Errington, R.D., Brown, J.S. and Vaughan, E.D. (1999) Survival and patterns of recurrence in 200 oral cancer patients treated by radical surgery and neck dissection. Oral Oncology, 35, 257-265. doi:10.1016/S1368-8375(98)00113-4

[3] Herranz González-Botas, J., Vázquez Barro, C., López Amado, M., Martínez Moran, A. and Chao Vieites, J. (2002) Factors affecting local and regional control and survival of carcinomas of the tongue and floor of mouth. Acta Otorrinolaringológica Española, 53, 32-38.

[4] Sutton, D.N., Brown, J.S., Rogers, S.N., Vaughan, E.D. and Woolgar, J.A. (2003) The prognostic implications of the surgical margin in oral squamous cell carcinoma. International Journal of Oral and Maxillofacial Surgery, 32, 30-34. doi:10.1054/ijom.2002.0313

[5] Koea, J.B. and Shaw, J.H. (1989) Cancer of the tongue and oral cavity in Auckland, New Zealand, 1970-1986. The Australian and New Zealand Journal of Surgery, 59, 39-45. doi:10.1111/j.1445-2197.1989.tb01463.x

[6] Looser, K.G., Shah, J.P. and Strong, E.W. (1978) The significance of "positive" margins in surgically resected epidermoid carcinomas. Head \& Neck Surgery, 1, 107111. doi:10.1002/hed.2890010203

[7] Jones, A.S. (1994) Prognosis in mouth cancer: Tumour factors. European Journal of Cancer, Part B, Oral Oncology, 30B, 8-15. doi:10.1016/0964-1955(94)90044-2

[8] Binahmed, A., Nason, R.W. and Abdoh, A.A. (2007) The clinical significance of the positive surgical margin in oral cancer. Oral Oncology, 43, 780-784. doi:10.1016/j.oraloncology.2006.10.001

[9] Haque, R., Contreras, R., McNicoll, M.P., Eckberg, E.C. and Petitti, D.B. (2006) Surgical margins and survival after head and neck cancer surgery. BMC Ear, Nose, and Throat Disorders, 6, 2. doi:10.1186/1472-6815-6-2

[10] Sessions, D.G., Spector, G.J., Lenox, J., Haughey, B., Chao, C. and Marks, J. (2002) Analysis of treatment results for oral tongue cancer. The Laryngoscope, 112, 616625. doi:10.1097/00005537-200204000-00005

[11] Lee, J.A. (2005) Datasets for reporting cancers datasets for histopathology reports on head and neck carcinomas and salivary neoplasms. 2nd Edition, Royal College of Pathologists, London.

[12] Johnson, R.E., Sigman, J.D., Funk, G.F., Robinson, R.A. and Hoffman, H.T. (1997) Quantification of surgical margin shrinkage in the oral cavity. Head \& Neck, 19, 281286.

doi:10.1002/(SICI)1097-0347(199707)19:4<281::AID-H ED6>3.3.CO;2-4

[13] Mistry, R.C., Qureshi, S.S. and Kumaran, C. (2005) Postresection mucosal margin shrinkage in oral cancer: Quantification and significance. Journal of Surgical Oncology, 91, 131-133. doi:10.1002/jso.20285

[14] Yuen, P.W., Lam, K.Y., Chan, A.C., Wei, W.I. and Lam, L.K. (1998) Clinicopathological analysis of local spread of carcinoma of the tongue. American Journal of Surgery, 175, 242-244. doi:10.1016/S0002-9610(97)00282-1

[15] Kakarala, K., Faquin, W.C. and Deschler, D.G. (2010) A comparison of histopathologic margin assessment after steel scalpel, monopolar electrosurgery, and ultrasonic scalpel glossectomy in a rat model. The Laryngoscope, 120, S155. doi:10.1002/lary.21619

[16] Spiro, R.H., Guillamondegui, O., Paulino, A.F. and Huvos, A.G. (1999) Pattern of invasion and margin assessment in patients with oral tongue cancer. Head \& Neck, 21, 408-413.

doi:10.1002/(SICI)1097-0347(199908)21:5<408::AID-H ED5>3.0.CO;2-E

[17] Byers, R.M., Bland, K.I., Borlase, B. and Luna, M. (1978) The prognostic and therapeutic value of frozen section determinations in the surgical treatment of squamous carcinoma of the head and neck. American Journal of Surgery, 136, 525-528. doi:10.1016/0002-9610(78)90275-1

[18] Scholl, P., Byers, R.M., Batsakis, J.G., Wolf, P. and Santini, H. (1986) Microscopic cut-through of cancer in the surgical treatment of squamous carcinoma of the tongue. Prognostic and therapeutic implications. American Journal of Surgery, 152, 354-360. doi:10.1016/0002-9610(86)90304-1

[19] Gauthier, P., Audet, N., Guertin, L., Arteau-Gauthier, I., Comeau, L., Pilon, L., et al. (2010) Complete frozen section margins (with measurable 1 or $5 \mathrm{~mm}$ thick free margin) for cancer of the tongue: Part 2: Clinical experience. Journal of Otolaryngology-Head \& Neck Surgery, 39, 20-27.

[20] Songra, A.K., Ng, S.Y., Farthing, P., Hutchison, I.L. and Bradley, P.F. (2006) Observation of tumour thickness and resection margin at surgical excision of primary oral squamous cell carcinoma-Assessment by ultrasound. International Journal of Oral and Maxillofacial Surgery, 35, 324-331. doi:10.1016/j.ijom.2005.07.019

[21] Tominaga, K., Yamamoto, K., Khanal, A., Morimoto, Y., Tanaka, T., Kodama, M., et al. (2007) Intraoperative surgical clearance confirmation of tongue carcinomas using ultrasound. Dento Maxillo Facial Radiology, 36, 409-411. doi:10.1259/dmfr/95685944

[22] Umeda, M., Shigeta, T., Takahashi, H., Minamikawa, T., Komatsubara, H., Oguni, A., et al. (2011) Clinical evaluation of Lugol's iodine staining in the treatment of stage I-II squamous cell carcinoma of the tongue. International Journal of Oral and Maxillofacial Surgery, 40, 593-596. doi:10.1016/j.ijom.2010.11.026

[23] Kurita, H., Sakai, H., Kamata, T., Koike, T., Kobayashi, 
H. and Kurashina, K. (2008) Accuracy of intraoperative tissue staining in delineating deep surgical margins in oral carcinoma surgery. Oral Oncology, 44, 935-940. doi:10.1016/j.oraloncology.2007.12.008

[24] Keereweer, S., Kerrebijn, J.D.F., Mol, I.M., Mieog, J.S.D., Van Driel, P.B.A., Baatenburg de Jong, R.J., et al. (2011) Optical imaging of oral squamous cell carcinoma and cervical lymph node metastasis. Head \& Neck, 34, 1002-1008.

[25] Lee, C.-C., Ho, H.-C. and Chou, P. (2010) Multivariate analyses to assess the effect of surgeon volume on survival rate in oral cancer: A nationwide population-based study in Taiwan. Oral Oncology, 46, 271-275. doi:10.1016/j.oraloncology.2010.01.006

[26] McMahon, J.D., Devine, J.C., Wong, L.S., Wales, C., Smith, M., James, A., et al. (2012) Anatomical surgical planning for oral and oropharyngeal primary carcinoma combined with adjuvant treatment where indicated is associated with improved local control. The British Journal of Oral \& Maxillofacial Surgery, 50, 706-711. doi:10.1016/j.bjoms.2012.02.009

[27] McMahon, J.D., Devine, J.C., Hetherington, J., Bryson, G., Mclellan, D., Maciver, C., et al. (2011) Involved surgical margins in oral and oropharyngeal carcinoma-An anatomical problem? The British Journal of Oral \& Maxillofacial Surgery, 49, 172-175.

doi:10.1016/j.bjoms.2010.02.014

[28] Batsakis, J.G. (1999) Surgical excision margins: A pathologist's perspective. Advances in Anatomic Pathology, 6, 140-148. doi:10.1097/00125480-199905000-00002

[29] Wong, L.S., McMahon, J., Devine, J., McLellan, D., Thompson, E., Farrow, A., et al. (2012) Influence of close resection margins on local recurrence and diseasespecific survival in oral and oropharyngeal carcinoma. The British Journal of Oral \& Maxillofacial Surgery, 50, 102-108. doi:10.1016/j.bjoms.2011.05.008

[30] Brandwein-Gensler, M., Teixeira, M.S., Lewis, C.M., Lee, B., Rolnitzky, L., Hille, J.J., et al. (2005) Oral squamous cell carcinoma: Histologic risk assessment, but not margin status, is strongly predictive of local disease-free and overall survival. The American Journal of Surgical Patheology, 29, 167-178. doi:10.1097/01.pas.0000149687.90710.21

[31] Partridge, M., Li, S.R., Pateromichelakis, S., Francis, R., Phillips, E., Huang, X.H., et al. (2000) Detection of minimal residual cancer to investigate why oral tumors recur despite seemingly adequate treatment. Clinical Cancer Research, 6, 2718-2725. 